\title{
A systematic review of next-generation point-of-care stroke diagnostic technologies
}

\author{
Shane Shahrestani, PhD, MS, ${ }^{1,2}$ Danielle Wishart, BA, ${ }^{1}$ Sung Min J. Han, MS, ${ }^{1}$ \\ Ben A. Strickland, MD, ${ }^{1}$ Joshua Bakhsheshian, MD, ${ }^{1}$ William J. Mack, MD, ${ }^{1}$ Arthur W. Toga, PhD, ${ }^{3}$ \\ Nerses Sanossian, MD, ${ }^{4}$ Yu-Chong Tai, PhD, ${ }^{2}$ and Gabriel Zada, MD, MS ${ }^{1}$
}

Departments of ${ }^{1}$ Neurological Surgery and ${ }^{4}$ Neurology, Keck School of Medicine, University of Southern California, Los Angeles; 2Department of Medical Engineering, California Institute of Technology, Pasadena; and ${ }^{3}$ Laboratory of Neurolmaging (LONI), USC Stevens Neuroimaging and Informatics Institute, Keck School of Medicine, University of Southern California, Los Angeles, California

OBJECTIVE Stroke is a leading cause of morbidity and mortality. Current diagnostic modalities include CT and MRI. Over the last decade, novel technologies to facilitate stroke diagnosis, with the hope of shortening time to treatment and reducing rates of morbidity and mortality, have been developed. The authors conducted a systematic review to identify studies reporting on next-generation point-of-care stroke diagnostic technologies described within the last decade.

METHODS A systematic review was performed according to PRISMA guidelines to identify studies reporting noninvasive stroke diagnostics. The QUADAS-2 (Quality Assessment of Diagnostic Accuracy Studies-2) tool was utilized to assess risk of bias. PubMed, Web of Science, and Scopus databases were utilized. Primary outcomes assessed included accuracy and timing compared with standard imaging, potential risks or complications, potential limitations, cost of the technology, size/portability, and range/size of detection.

RESULTS Of the 2646 reviewed articles, 19 studies met the inclusion criteria and included the following modalities of noninvasive stoke detection: microwave technology (6 studies, 31.6\%), electroencephalography (EEG; 4 studies, $21.1 \%$ ), ultrasonography (3 studies, 15.8\%), near-infrared spectroscopy (NIRS; 2 studies, 10.5\%), portable MRI devices (2 studies, 10.5\%), volumetric impedance phase-shift spectroscopy (VIPS; 1 study, 5.3\%), and eddy current damping (1 study, $5.3 \%$ ). Notable medical devices that accurately predicted stroke in this review were EEG-based diagnosis, with a maximum sensitivity of $91.7 \%$ for predicting a stroke, microwave-based diagnosis, with an area under the receiver operating characteristic curve (AUC) of 0.88 for differentiating ischemic stroke and intracerebral hemorrhage (ICH), ultrasound with an AUC of 0.92, VIPS with an AUC of 0.93 , and portable MRI with a diagnostic accuracy similar to that of traditional MRI. NIRS offers significant potential for more superficially located hemorrhage but is limited in detecting deep-seated ICH (2.5-cm scanning depth).

CONCLUSIONS As technology and computational resources have advanced, several novel point-of-care medical devices show promise in facilitating rapid stroke diagnosis, with the potential for improving time to treatment and informing prehospital stroke triage.

https://thejns.org/doi/abs/10.3171/2021.4.FOCUS21122

KEYWORDS eddy current damping; near-infrared spectroscopy; volumetric impedance phase-shift spectroscopy; microwave; ischemic stroke; hemorrhagic stroke

$\mathrm{S}$ TROKE represents the fifth most common cause of mortality within the United States, with approximately one person dying of stroke every $3.6 \mathrm{~min}-$ utes. ${ }^{1}$ There continues to be an emphasis on accurate and rapid detection, as time to treatment is paramount in optimizing outcomes. The timing of intervention within acute ischemic stroke care is critical, with national guidelines calling for a door-to-treatment time of less than 1 hour. ${ }^{2,3}$ Several factors may contribute toward delays in stroke diagnosis and treatment. Two established contributors are prolonged stroke imaging ( $>20$ minutes) and complicated triage/transport by emergency medical services delaying imaging. ${ }^{4}$ Both of these factors stem in part from limitations of currently available diagnostic technologies,

ABBREVIATIONS AUC = area under the receiver operating characteristic curve; $E C D=$ eddy current damping; $E E G=$ electroencephalography; $I C H=$ intracerebral hemorrhage; NIRS = near-infrared spectroscopy; QUADAS-2 = Quality Assessment of Diagnostic Accuracy Studies-2; VIPS = volumetric impedance phase-shift spectroscopy. SUBMITTED February 26, 2021. ACCEPTED April 8, 2021.

INCLUDE WHEN CITING DOI: 10.3171/2021.4.FOCUS21122. 


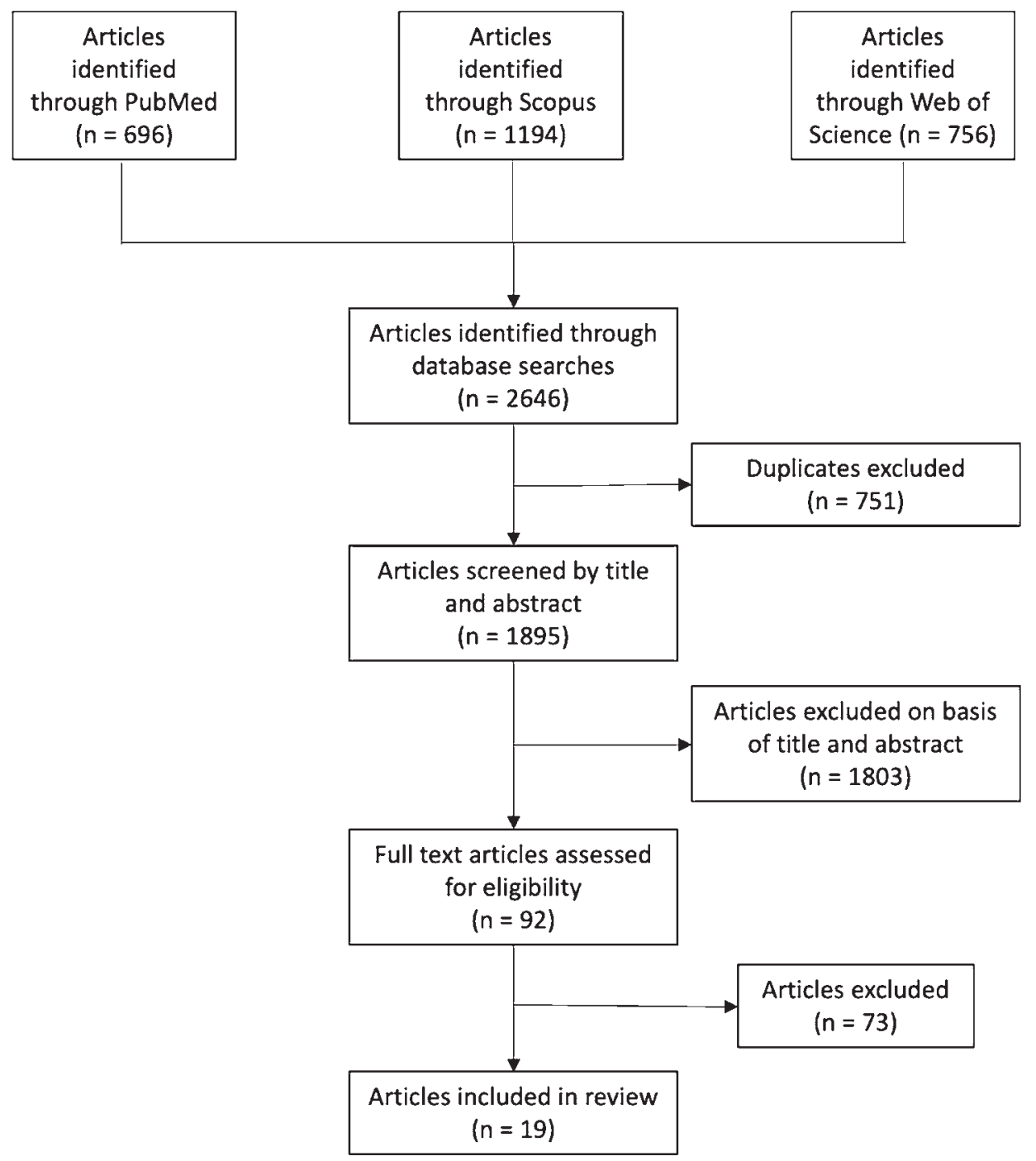

FIG. 1. PRISMA flowchart outlining the search and review process used to identify and select articles for inclusion in this systematic review.

including the lack of portability of CT and MRI, which heavily depends on factors such as hospital crowding and geographic location..$^{5,6}$

To address these shortcomings, several novel technologies and approaches have been developed to facilitate the diagnosis of stroke, with the hope of shortening the time to treatment and reducing the rates of morbidity and mortality. These approaches vary widely and include microwave-based analysis, volumetric impedance phaseshift spectroscopy (VIPS), near-infrared spectroscopy (NIRS), electroencephalography (EEG), transcranial Doppler ultrasound, and eddy current damping (ECD), among others. ${ }^{7-14}$ Several of these technologies have already progressed to clinical trials, and some have gained clearance from the FDA. ${ }^{8,15}$ Despite major differences in operational principles, all of these newly emerging technologies share a similar end goal: to develop portable diagnostic technology in an effort to more efficiently triage stroke care. ${ }^{7-14}$
As stroke diagnostic technology continues to progress and novel technological avenues are discovered, it is imperative to assess the efficacy of these next-generation medical devices to ensure that the most efficient and accurate diagnostic and treatment plans are utilized. In this study, we conducted a contemporary systematic review of the current state of point-of-care stroke detection.

\section{Methods}

\section{Search Strategy}

In February 2021, a search of the PubMed, Web of Science, and Scopus databases in accordance with the Preferred Reporting Items for Systematic Reviews and Meta-Analyses (PRISMA) guidelines (Fig. 1) was conducted. To identify studies reporting novel stroke diagnostic techniques, we used the following Boolean search terms: ((stroke OR hemorrhagic stroke OR thrombotic stroke OR ischemic stroke OR embolic stroke) AND ((di- 
TABLE 1. Summary of QUADAS-2 tool assessment for all reviewed studies

\begin{tabular}{|c|c|c|c|c|}
\hline $\begin{array}{l}\text { Authors } \\
\text { \& Year }\end{array}$ & $\begin{array}{c}\text { Risk of Bias: } \\
\text { Patient Selection }\end{array}$ & $\begin{array}{c}\text { Risk of Bias: Evaluation } \\
\text { of Index Test }\end{array}$ & $\begin{array}{l}\text { Risk of Bias: Evaluation } \\
\text { of Reference Standard }\end{array}$ & $\begin{array}{l}\text { Risk of Bias: } \\
\text { Flow \& Timing }\end{array}$ \\
\hline FDA, $2010^{15}$ & Unclear & Low & High & Unclear \\
\hline Abtahi et al., $2012^{7}$ & Unclear & Low & High & Unclear \\
\hline Schlachetzki et al., $2012^{26}$ & Low & Low & Low & High \\
\hline Persson et al., $2014^{11}$ & Unclear & Low & High & Unclear \\
\hline Michelson et al., $2015^{23}$ & Low & High & High & Low \\
\hline Mobashsher et al., $2016^{14}$ & Low & High & High & Low \\
\hline Brogan et al., $2017^{9}$ & Low & Low & Low & High \\
\hline Bashri et al., $2017^{21}$ & Low & Low & Low & Low \\
\hline Kellner et al., $2018^{8}$ & High & Low & Low & High \\
\hline Thorpe et al., $2019^{27}$ & High & High & High & Unclear \\
\hline Coli et al., $2019^{22}$ & Low & High & Low & Low \\
\hline Shreve et al., $2019^{13}$ & Low & Low & Low & Low \\
\hline Alqadami et al., $2020^{20}$ & Low & Low & Low & High \\
\hline Cooley et al., $2021^{29}$ & Low & Low & Low & Unclear \\
\hline Gottlibe et al., $2020^{10}$ & Low & Low & Low & Low \\
\hline Guasch et al., $2020^{25}$ & Low & Low & Low & Low \\
\hline Shahrestani et al., $2020^{12}$ & Low & High & Low & Unclear \\
\hline Sheth et al., $2021^{28}$ & Low & Low & Low & High \\
\hline Wilkinson et al., $2020^{24}$ & Low & Low & Low & High \\
\hline
\end{tabular}

agnos* OR detection) AND (tool OR tech* OR device OR instrument OR machine)) AND (portable OR mobile OR compact OR point-of-care OR prehospital OR rapid)). The same search terms were used for each database, and syntax was adjusted accordingly. The reference lists of all included studies were also reviewed. Two authors (D.W. and S.M.J.H.) independently reviewed each article, and any discrepancies were discussed by an arbitrator (S.S.) until consensus was reached. D.W. and S.M.J.H. performed data extraction once the list of included studies was finalized.

\section{Selection Criteria and Data Collection}

Overall, the initial search identified 2646 studies. Removal of duplicates yielded 1895 studies, after which the following search criteria were applied: 1) studies describing stroke diagnostic technologies or devices other than traditional CT or MRI, 2) studies published within the last 10 years, and 3) studies written in the English language. The full text was reviewed if any discrepancies arose while parsing through the studies. This process yielded a total of 92 studies. Studies were excluded if they 1) involved conventional methods such as CT or MRI, 2) were book chapters, 3) were animal and/or nonhuman models, or 4) demonstrated biomarker detection of stroke (rather than medical device development). Nineteen studies met the inclusion and exclusion criteria and were included in the systematic review analysis. Outcomes were prespecified and included the cost of the technology, accuracy and timing compared with standard imaging, potential risks or complications, potential limitations, size/portability, and range/size of detection.

\section{Risk of Bias Analysis}

The Quality Assessment of Diagnostic Accuracy Studies-2 (QUADAS-2) tool was used to assess risk of bias (Table 1). This assessment tool is recommended by the Agency for Healthcare Research and Quality, the Cochrane Collaboration, and the United Kingdom National Institute for Health and Clinical Excellence. The QUADAS-2 itself encapsulates the following four metrics that may increase study bias: 1) patient selection, 2) evaluation of index tests, 3) evaluation of reference standards, and 4) flow and timing. ${ }^{16-19}$ Based on several yes/no questions, each of these metrics is then ranked as high risk of bias, low risk of bias, or unclear. The risk of bias was assessed independently by S.S., D.W., and S.M.J.H. for this systematic review, and any discrepancies were discussed until a consensus was reached.

\section{Results}

\section{Included Diagnostic Stroke Platforms}

Of the 19 studies identified through our systematic review, 6 implemented microwave technology (31.6\%), 4 used EEG (21.1\%), 3 used ultrasonography (15.8\%), 2 used NIRS (10.5\%), 2 used portable MRI devices (10.5\%), 1 used VIPS (5.3\%), and 1 used ECD (5.3\%) (Fig. 2). A summary of each study, including the diagnostic tool used, accuracy, and advantages or limitations of the tool are included in Table 2.

\section{Stroke Diagnosis Using Microwaves}

Several methods of nonionizing stroke detection are under investigation. The most popular medical device being 


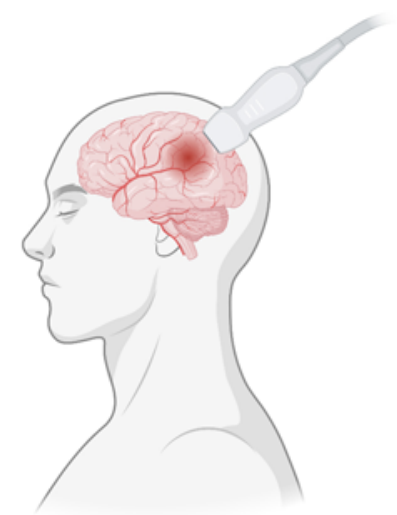

Ultrasound

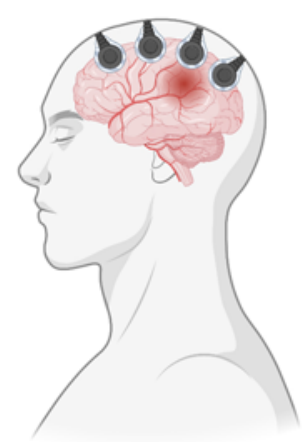

EEG

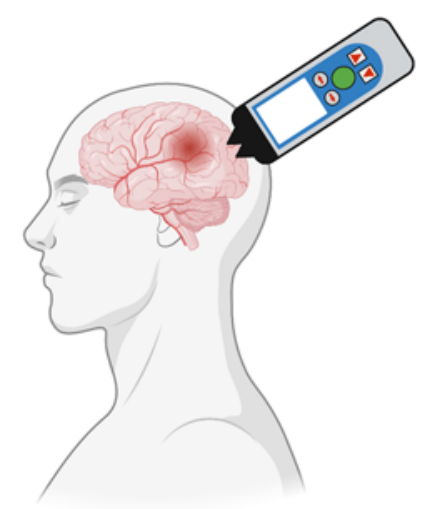

NIRS

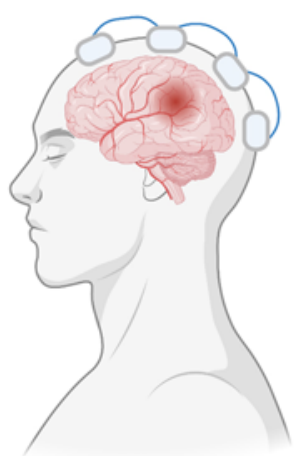

Microwave
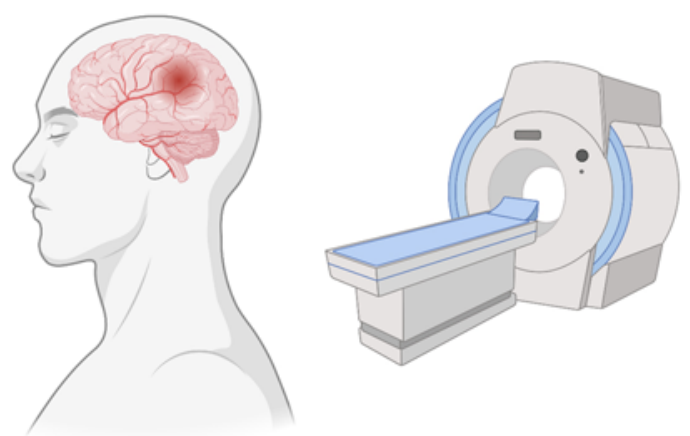

\section{Portable MRI}

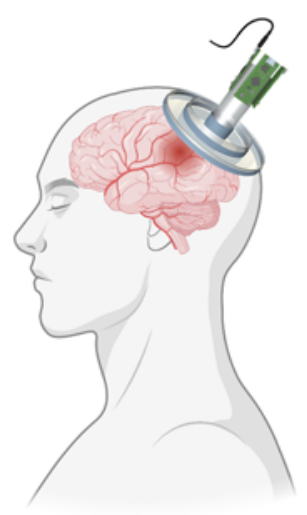

ECD

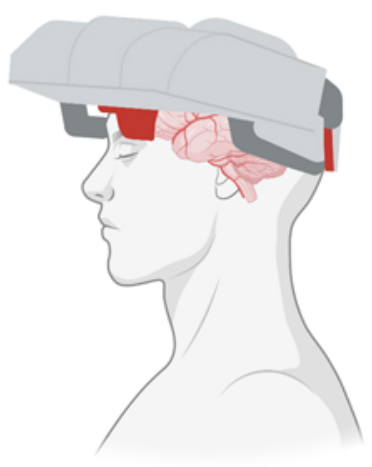

VIPS

FIG. 2. Schematic demonstrating the use of each novel stroke technology. Made with a valid license in @BioRender - biorender. com.

investigated for stroke diagnosis involves microwave-based stroke sensing. 7,11,14,20-22 This method was first described by Abtahi et al. in 2012. 'All of the studies investigating microwave technology underscored the low-cost, portability, and rapid diagnostic time of this method. ${ }^{7}$ In addition, this technology is good at distinguishing between ischemic and hemorrhagic stroke subtypes (Table 3$).{ }^{11}$ However, several limitations need to be addressed as they pertain to these sensors. First, the interface between the sensor and the head is an important consideration to maximize radiofrequency transmission through the skull. Abtahi et al. utilized a water bolus between the antenna and head to ensure signal transduction and described signal attenuation and leakage into free space instead of transmission into the head when using this technique. ' Similarly, the current spatial and depth resolution of microwave imaging is subpar and requires improvement. Several studies have noted that, at their current capacity, microwave-based technologies are unable to solely dictate thrombolytic therapy because small targets may not actually be detected..$^{11,14}$ One method of addressing the problem of resolution is by multiplexing microwave sensors and placing several on the head; Bashri et al. noted that the accuracy of microwave systems are dependent on the number of antennas. ${ }^{21}$ However, in exchange for increased accuracy in spatial resolution comes the trade-off of increased device size and complexity. In addition, Alqa- dami et al. reported that a maximum of 32 antennas can be placed to maximize the accuracy of microwave-based stroke detection devices. ${ }^{20}$ Microwave-based sensors hold significant future promise in rapid stroke detection if these limitations can be overcome.

\section{Stroke Diagnosis Using EEG}

Four studies successfully described EEG for stroke detection. The first study was published by Michelson et al. in 2015 and demonstrated acute stroke diagnosis (ischemic and hemorrhagic) with high sensitivity (91.7\%) and moderate specificity $(50.4 \%) .^{23}$ Further experimentation by Gottlibe et al., Shreve et al., and Wilkinson et al. confirmed the efficacy of EEG for ischemic stroke diagnosis using the changes in the revised brain symmetry index, alpha/delta frequency band ratios, and mixed delta/alpha ratio plus pairwise-derived brain symmetry index values, respectively. ${ }^{10,13,24}$ These studies suggested that EEG may accurately predict acute stroke within 3 minutes. In addition, EEG may be able to differentiate between hemorrhagic and ischemic stroke subtypes, since it has been shown to have an overall sensitivity of $91.7 \%$ for predicting any stroke, sensitivity of $90.3 \%$ for ischemic stroke, and sensitivity of $94.1 \%$ for hemorrhagic stroke. However, the main limitation of EEG detection is the massive setup 


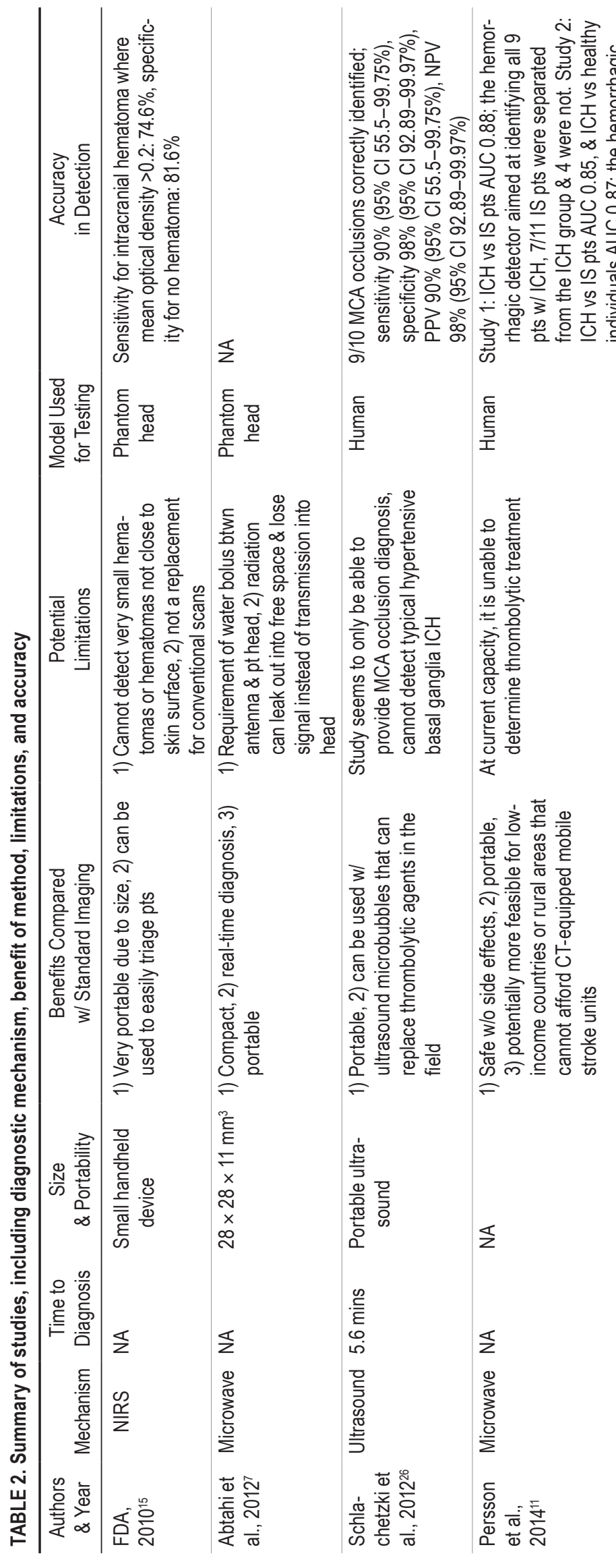

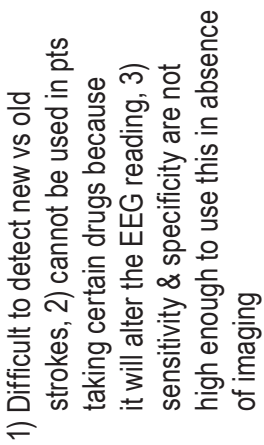

楆 产

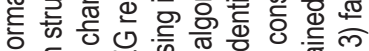

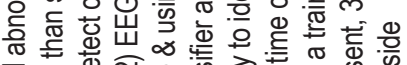

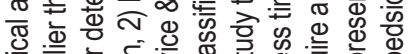

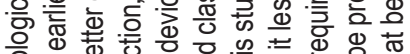

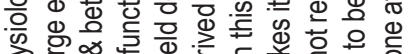

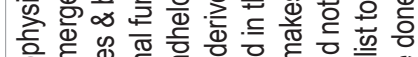

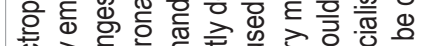

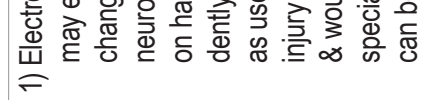

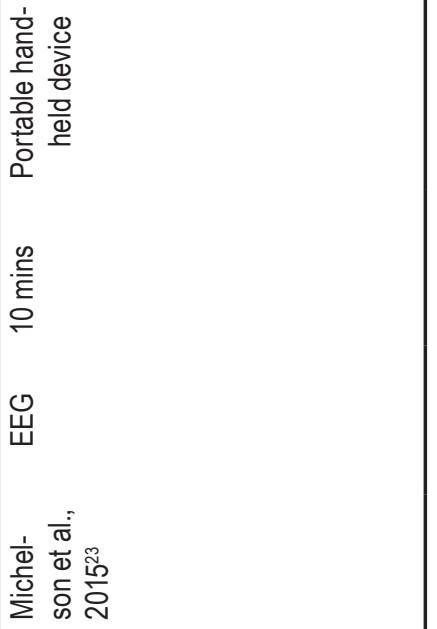




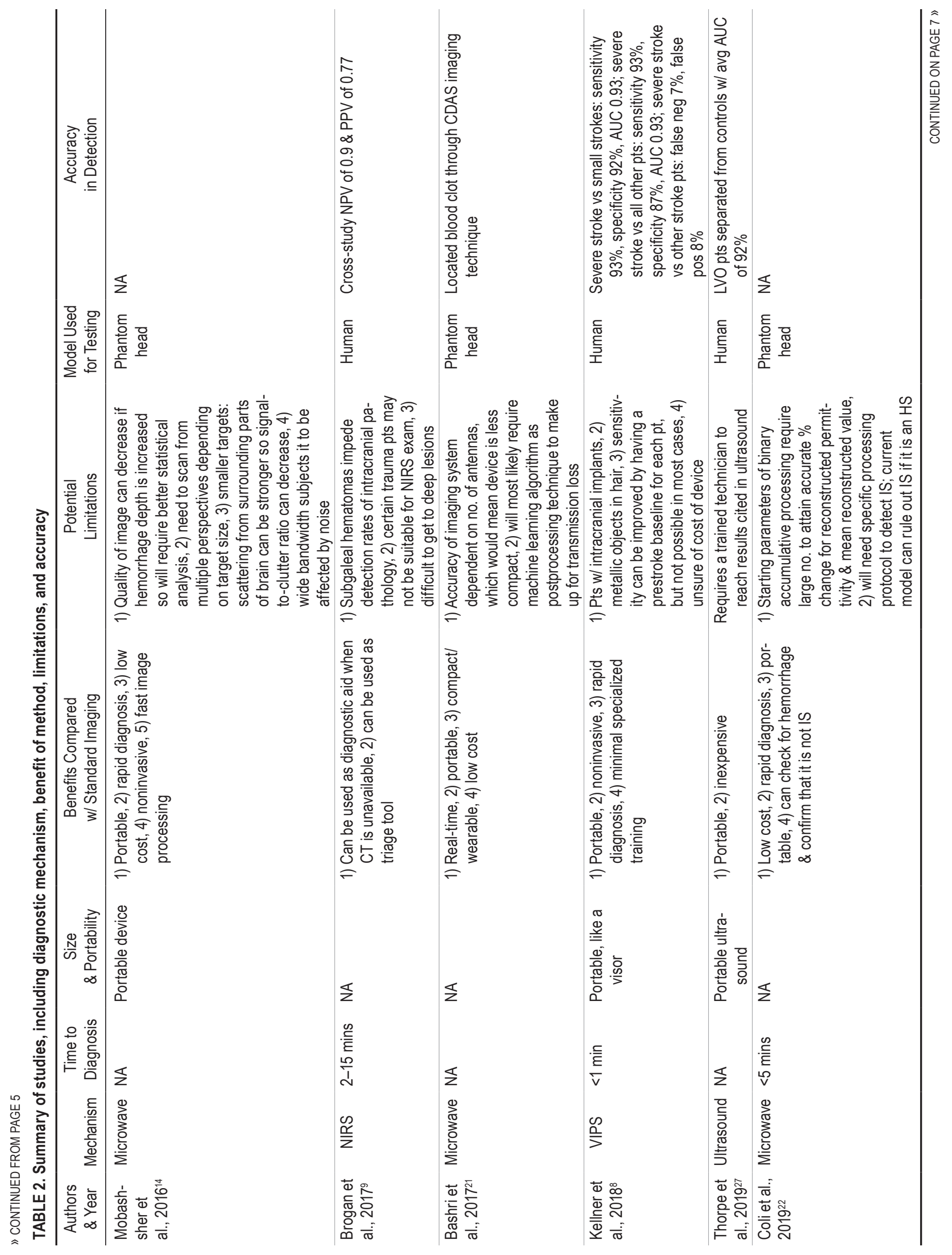



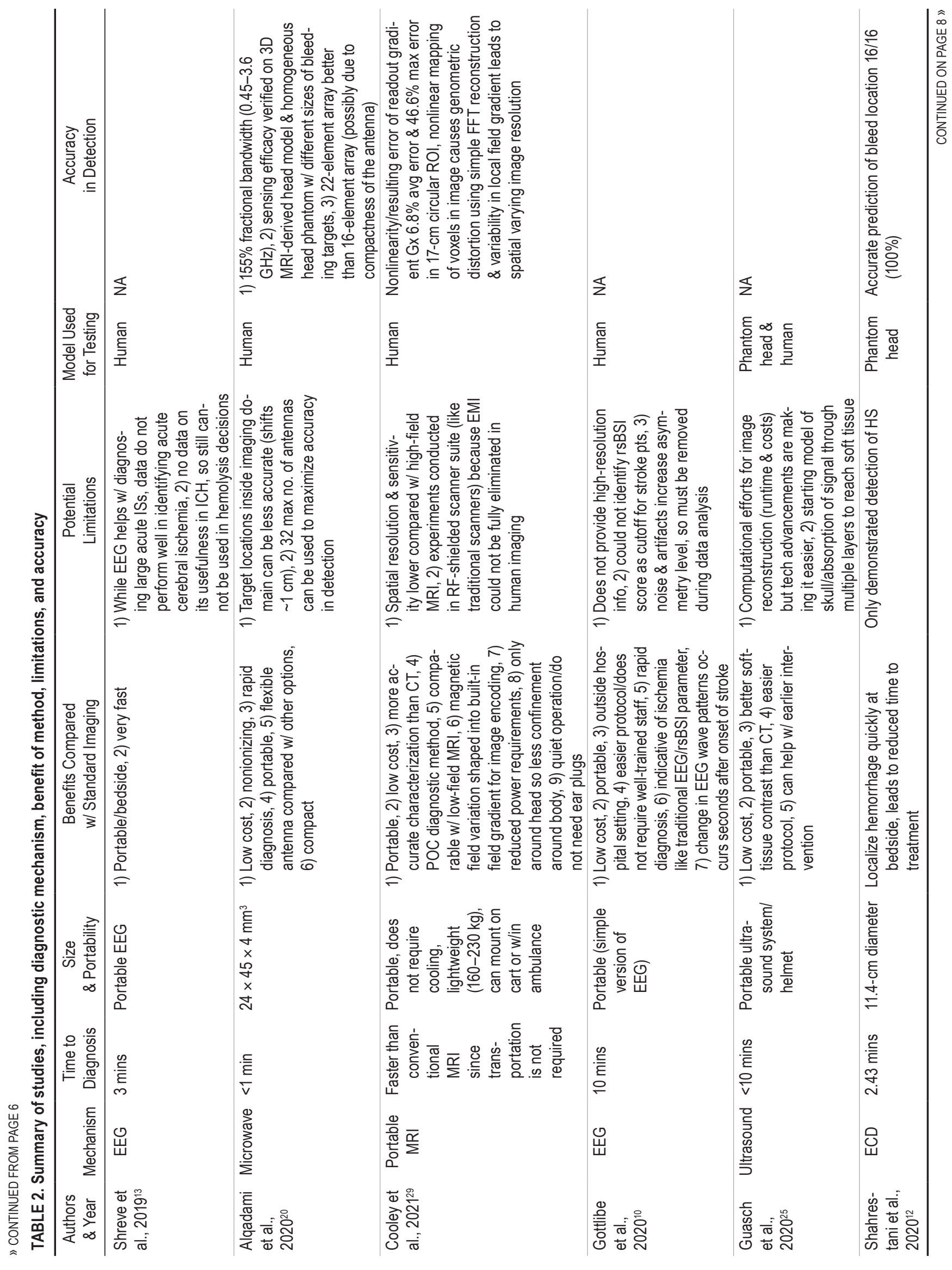

Neurosurg Focus Volume 51 • July 2021 


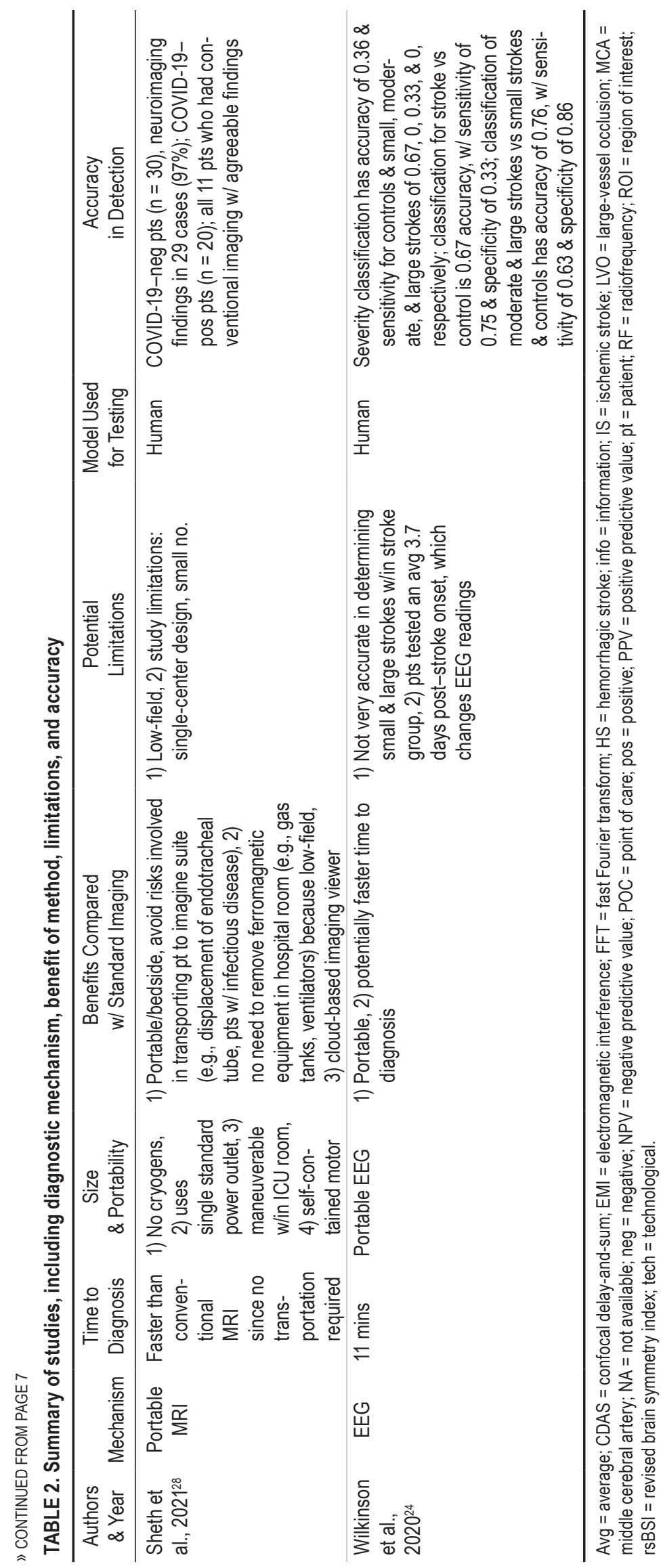


TABLE 3. Overview of stroke diagnostic technology included in this systematic review

\begin{tabular}{|c|c|c|c|c|}
\hline $\begin{array}{l}\text { Name of } \\
\text { Technology }\end{array}$ & $\begin{array}{l}\text { Description } \\
\text { of Technology }\end{array}$ & $\begin{array}{l}\text { Potential to } \\
\text { Differentiate IS vs HS }\end{array}$ & $\begin{array}{l}\text { Potential to Differentiate } \\
\text { Stroke From Stroke Mimics }\end{array}$ & $\begin{array}{l}\text { Potential to Detect } \\
\text { LVO Stroke }\end{array}$ \\
\hline Microwave & $\begin{array}{l}\text { Measuring microwave scattering due to the dielectric } \\
\text { contrast of different tissue types; usually used in a } \\
\text { helmet-like device w/ antennas }\end{array}$ & Yes & Unknown & No \\
\hline EEG & Using brain electrical activity to detect acute stroke & Yes & $\begin{array}{l}\text { Not determined (specificity } \\
\text { of } 50.4 \% \text { to stroke mimic in } \\
\text { Michelson et al. }{ }^{23} \text { ) }\end{array}$ & Unknown \\
\hline Ultrasonography & $\begin{array}{l}\text { Using ultrasound tomography to provide a 3D image } \\
\text { of brain to diagnose acute stroke }\end{array}$ & No & Unknown & Yes \\
\hline NIRS & $\begin{array}{l}\text { Detects absorption of } 805 \text {-nm wavelength, which is } \\
\text { sensitive to blood vol }\end{array}$ & No & No & No \\
\hline Portable MRI & Portable, low-field MRI to detect acute stroke & Yes & Yes & Yes \\
\hline VIPS & $\begin{array}{l}\text { Uses array of low-energy radio waves to detect bio- } \\
\text { impedance of different tissue type \& fluid properties }\end{array}$ & Unknown & Yes & Yes \\
\hline ECD & $\begin{array}{l}\text { Measures changes in electrical conductivity in the } \\
\text { brain by creating microtesla magnetic fields }\end{array}$ & Yes & Unknown & Unknown \\
\hline
\end{tabular}

"Unknown" is used in instances in which the comparison had not been studied or had not been well reported on. "Not determined" is used in instances in which a comparison had been reported but did not have definitive results.

time normally associated with placing numerous leads on the head. Furthermore, if MRI is indicated, all magnetic leads must be taken off of the patient, which requires additional time. Additional limitations include poor spatial resolution and that small infarcts or hemorrhages may not be detected using this method.

\section{Stroke Diagnosis Using Ultrasonography}

Ultrasound diagnosis of stroke is a nonionizing technique that is currently being investigated by several groups. ${ }^{25-27}$ The primary limitation of this technique is the need for ultrasound transducer materials to minimize signal attenuation. Even with appropriate transduction materials, the density of the skull contributes to significant signal attenuation, and the temporal bone window is frequently used for intracranial ultrasound applications. ${ }^{26}$ Even when implementing these findings, heavy computation is critical in accurately utilizing ultrasound for stroke diagnosis. Thorpe et al. demonstrated the calculation of a velocity curvature index from cerebral blood flow velocity using a transcranial Doppler probe. ${ }^{27}$ This method yielded a maximum area under the receiver operating characteristic curve (AUC) of 0.94, which represents one of the highest metrics of device performance reported in the contemporary stroke diagnostic device literature.

\section{Stroke Diagnosis Using NIRS}

The FDA has approved the use of NIRS for detection of hemorrhagic stroke. ${ }^{915}$ Nonionizing NIRS to determine absorption of a 805-nm wavelength, which is sensitive to blood volume and not blood oxygen saturation. ${ }^{15}$ While this technology allows for rapid and compact scanning and has a cross-study sensitivity of $78 \%$, specificity of $90 \%$, positive predictive value of $77 \%$, and negative predictive value of $90 \%$, it has several major limitations. ${ }^{9}$ First, NIRS is unable to detect ischemic stroke and can only detect hemorrhagic stroke $>3.5 \mathrm{~mL}$. Furthermore, NIRS can only detect hematomas within the most superficial $2.5 \mathrm{~cm}$ of the head. As such, this method is not helpful for detecting deeper intracerebral hemorrhage (ICH) (such as some basal ganglia ICHs) and cannot yet distinguish between stroke subtypes to facilitate treatment. Additionally, the NIRS device is only probed at 8 unique points on the head and is not indicated for continuous scanning.

\section{Stroke Diagnosis Using Portable MRI}

Aside from novel diagnostic technologies, significant advances have taken place with regard to portable MRI scanners. Both Sheth et al. and Cooley et al. described these portable advancements, which boast many of the benefits of traditional MRI, including accurate neuroimaging and millimeter resolution..$^{28,29}$ However, portable MRI scanners have several limitations compared with the other stroke diagnostic devices, including much larger sizes, increased power requirements, and increased device costs, limiting widespread availability.

\section{Stroke Diagnosis Using VIPS}

Another nonionizing stroke diagnostic technique is VIPS, which uses bioimpedance asymmetry scores to predict large-vessel occlusions. ${ }^{8}$ While the VIPS device is portable, noninvasive, and easy to use and has a sensitivity of $93 \%$ and specificity of $92 \%$ for large-vessel stroke, it has not yet been shown to work effectively for differentiation of ischemic and hemorrhagic stroke. In addition, VIPS devices are extremely sensitive to metal (e.g., metallic implants, metal objects worn in the hair), and the presence of metal can significantly disrupt the signal.

\section{Stroke Diagnosis Using ECD}

ECD sensors represent a nonionizing stroke diagnostic 
technology. ${ }^{12}$ These sensors are $11 \mathrm{~cm}$ in diameter and operate by creating microtesla-level magnetic fields capable of detecting changes in electrical conductivity within the brain, with ischemia having reduced conductivity and hemorrhage having increased conductivity. Prior studies have demonstrated a scanning depth of $5 \mathrm{~cm}$ into the brain, with accurate (100\% detection) image production of hemorrhagic stroke within 2.43 minutes..$^{12}$ However, current limits of hemorrhage detection have been reported to be $25 \mathrm{~mL}$, and it has been shown to also be sensitive to the presence of metal objects.

\section{Discussion}

The present systematic review describes contemporary and evolving stroke diagnostic technologies, their proposed benefits and limitations, and current accuracy in diagnosis. Following our comprehensive review of the literature, we identified 7 diagnostic avenues that are currently being investigated or preliminarily implemented for the assessment of stroke. The primary benefit of next-generation stroke technology centers around portability. All of the studies included in this study emphasized the need for portable stroke-sensing capabilities to facilitate triage and save time compared with CT or MRI. Furthermore, rapid prehospital diagnosis (compared with traditional imaging) was emphasized as a potential benefit in all studies. A wide variety of limitations was also discussed for each diagnostic method. Most notably, scanning depth into the brain and the detection of submillimeter lesions require further investigation. In addition, VIPS and ECD sensors were highly sensitive to the presence of metal, which may be present as a medical implant or within patient clothing.

Recent technological advances have allowed for the development of technologies for stroke detection and have also allowed for the modification of preexisting diagnostic devices for stroke applications. Technologies such as ultrasonography and EEG are being retrofitted for stroke applications thanks to recent computational advancements, including finite element modeling and machine learning. ${ }^{24,30}$ One major benefit of the utilization of preexisting diagnostic tools for stroke diagnostics is the well-understood risks and benefits associated with the technology. Such a thorough understanding also greatly reduces the difficulty of obtaining FDA approval, allowing for a shortened timeline to market. Notable medical devices that accurately predicted stroke in our review included EEG-based diagnosis with a maximum sensitivity of $91.7 \%$ for predicting a stroke, ${ }^{23}$ microwave-based diagnosis with an AUC of 0.88 for differentiating ischemic stroke and $\mathrm{ICH},{ }^{11}$ ultrasound with an AUC of $0.92,{ }^{27}$ VIPS with an AUC of $0.93,{ }^{8}$ and portable MRI with a diagnostic accuracy similar to that of traditional MRI. ${ }^{28,29}$

In addition, current technologies require further innovation to accurately classify stroke subtypes while achieving acceptable scanning depths and volume sensitivity. As previously mentioned, FDA-approved NIRS methods are currently indicated only for suspected hemorrhagic stroke and cannot distinguish ischemic and hemorrhagic stroke subtypes. ${ }^{9115}$ Furthermore, the moderate sensitivity of NIRS, volume limits, and detection range prevent it from being useful in cases of suspected deep or small ICHs. ${ }^{915}$ Similarly, microwave-based methods and EEG have also been described to have a limited ability for detection of small hematomas, which prevents their use for high-accuracy stroke subtyping and for the guidance of thrombolytic administration. . $11,11,14,24$ The technology that shows the greatest potential promise for the detection and classification of stroke subtypes is currently portable MRI, which essentially operates as a miniaturized compact version of traditional MRI. However, these devices are much more expensive and much larger than comparable nextgeneration stroke diagnostic technologies. ${ }^{28,29}$ Further research on VIPS and ECD methods for stroke detection are necessary to fully understand their capabilities in ischemic and hemorrhagic stroke detection and classification. ${ }^{8}$

With the hope of further improving the detection of stroke, biomarker approaches are also being investigated but remain outside of the scope of this review. One of the best biomarker diagnostic performances described in the literature was achieved when using apolipoprotein A1unique peptide as a biomarker for acute ischemic stroke, with an AUC of 0.975 , a sensitivity of $90.63 \%$, and a specificity of $97.14 \% .{ }^{31}$ Similarly, Tao et al. conducted a study with the second largest AUC of 0.879, which was achieved by combining lipoprotein-associated phospholipase A2, serum amyloid A, and fibrinogen as diagnostic biomarkers for acute cerebral infarction. ${ }^{32}$ However, among all of the blood-based biomarker diagnostic tests, one major problem remains: access to a laboratory for the analysis of blood components. This is a huge limitation, and laboratory processing times may take several additional hours depending on the assay required for diagnosis (e.g., enzyme-linked immunosorbent assay).

\section{Limitations}

There are several limitations to this study. First, many of the technologies described in this article are exploratory, and additional multicenter randomized controlled studies are necessary to fully confirm the efficacy of these stroke diagnostic methodologies. Second, significant heterogeneity existed within each novel diagnostic subgroup discussed in our review. This is likely due to the fact that optimal protocols have not yet been established because of the experimental nature of current investigations. Third, several of the devices discussed herein were specific for either ischemic or hemorrhagic stroke, and significant innovation is still required to differentiate the two subtypes.

\section{Conclusions}

As technology and computational resources have advanced, several next-generation medical devices have emerged that show promise in facilitating stroke diagnosis. These improvements include reduced diagnostic times, increased device compactness allowing for portable diagnosis, and reduced device costs. As additional data continue to emerge regarding these novel methods, it is imperative to reexamine the current stroke diagnostic paradigm and updated guidelines for diagnosis and management appropriately. Overall, additional studies are necessary to thoroughly investigate the benefits and limitations of emerging 
stroke diagnostic technologies with the hope of improving stroke management and reducing rates of mortality and morbidity associated with patient transport or imaging availability.

\section{References}

1. Virani SS, Alonso A, Benjamin EJ, et al. Heart disease and stroke statistics - 2020 update: a report from the American Heart Association. Circulation. 2020;141(9):e139-e596.

2. Kelly AG, Hellkamp AS, Olson D, et al. Predictors of rapid brain imaging in acute stroke: analysis of the Get With the Guidelines-Stroke program. Stroke. 2012;43(5):1279-1284.

3. Jauch EC, Saver JL, Adams HP Jr, et al. Guidelines for the early management of patients with acute ischemic stroke: a guideline for healthcare professionals from the American Heart Association/American Stroke Association. Stroke. 2013:44(3):870-947.

4. Mowla A, Doyle J, Lail NS, et al. Delays in door-to-needle time for acute ischemic stroke in the emergency department: a comprehensive stroke center experience. J Neurol Sci. 2017; 376:102-105.

5. Reznek MA, Murray E, Youngren MN, et al. Door-toimaging time for acute stroke patients is adversely affected by emergency department crowding. Stroke. 2017;48(1): 49-54.

6. Adeoye O, Albright KC, Carr BG, et al. Geographic access to acute stroke care in the United States. Stroke. 2014;45(10): 3019-3024.

7. Abtahi S, Yang J, Kidborg S. A new compact multiband antenna for stroke diagnosis system over $0.5-3 \mathrm{GHz}$. Microw Opt Technol Lett. 2012;54(10):2342-2346.

8. Kellner CP, Sauvageau E, Snyder KV, et al. The VITAL study and overall pooled analysis with the VIPS non-invasive stroke detection device. J Neurointerv Surg. 2018;10(11): 1079-1084.

9. Brogan RJ, Kontojannis V, Garara B, et al. Near-infrared spectroscopy (NIRS) to detect traumatic intracranial haematoma: a systematic review and meta-analysis. Brain Inj. 2017; 31(5):581-588.

10. Gottlibe M, Rosen O, Weller B, et al. Stroke identification using a portable EEG device - a pilot study. Neurophysiol Clin. 2020;50(1):21-25.

11. Persson M, Fhager A, Trefná HD, et al. Microwave-based stroke diagnosis making global prehospital thrombolytic treatment possible. IEEE Trans Biomed Eng. 2014;61(11): 2806-2817.

12. Shahrestani S, Chou TC, Tai YC. A portable and rapid stroke imaging and classification device. Stroke. 2020;51(Suppl1): WP288.

13. Shreve L, Kaur A, Vo C, et al. Electroencephalography measures are useful for identifying large acute ischemic stroke in the emergency department. J Stroke Cerebrovasc Dis. 2019; 28(8):2280-2286.

14. Mobashsher AT, Bialkowski KS, Abbosh AM, Crozier S. Design and experimental evaluation of a non-invasive microwave head imaging system for intracranial haemorrhage detection. PLoS One. 2016;11(4):e0152351.

15. Food and Drug Administration. De novo classification request for Infrascan, Inc.'s Infrascanner Model 1000. April 2010. Accessed May 7, 2021. https://www.accessdata.fda.gov/ cdrh_docs/reviews/K080377.pdf

16. Nguyen AV, Blears EE, Ross E, et al. Machine learning applications for the differentiation of primary central nervous system lymphoma from glioblastoma on imaging: a systematic review and meta-analysis. Neurosurg Focus. 2018;45(5):E5.

17. Whiting PF, Rutjes AWS, Westwood ME, et al. QUADAS-2: a revised tool for the quality assessment of diagnostic accuracy studies. Ann Intern Med. 2011;155(8):529-536.
18. Santaguida PL, Riley CM, Matchar DB. Chapter 5: assessing risk of bias as a domain of quality in medical test studies. $J$ Gen Intern Med. 2012;27(suppl 1):S33-S38.

19. Reitsma JB, Rutjes AWS, Whiting P, et al. Assessing Methodological Quality. The Cochrane Collaboration; 2009.

20. Alqadami ASM, Stancombe AE, Bialkowski KS, Abbosh A. Flexible meander-line antenna array for wearable electromagnetic head imaging. IEEE Trans Antennas Propag. Published online November 18, 2020. doi:10.1109/ TAP.2020.3037742

21. Bashri MSR, Arslan T. Low-cost and compact RF switching system for wearable microwave head imaging with performance verification on artificial head phantom. IET Microw Antennas Propag. 2017;12(5):706-711.

22. Coli VL, Tournier P, Dolean V, et al. Detection of simulated brain strokes using microwave tomography. IEEE J Electromagn RF Microw Med Biol. 2019;3(4):254-260.

23. Michelson EA, Hanley D, Chabot R, Prichep LS. Identification of acute stroke using quantified brain electrical activity. Acad Emerg Med. 2015;22(1):67-72.

24. Wilkinson CM, Burrell JI, Kuziek JWP, et al. Predicting stroke severity with a 3-min recording from the Muse portable EEG system for rapid diagnosis of stroke. Sci Rep. 2020;10(1):18465.

25. Guasch L, Calderón Agudo O, Tang MX, et al. Full-waveform inversion imaging of the human brain. NPJ Digit Med. 2020;3:28.

26. Schlachetzki F, Herzberg M, Hölscher T, et al. Transcranial ultrasound from diagnosis to early stroke treatment: part 2: prehospital neurosonography in patients with acute stroke: the Regensburg stroke mobile project. Cerebrovasc Dis. 2012;33(3):262-271.

27. Thorpe SG, Thibeault CM, Wilk SJ, et al. Velocity curvature index: a novel diagnostic biomarker for large vessel occlusion. Transl Stroke Res. 2019;10(5):475-484.

28. Sheth KN, Mazurek MH, Yuen MM, et al. Assessment of brain injury using portable, low-field magnetic resonance imaging at the bedside of critically ill patients. JAMA Neurol. 2021;78(1):41-47.

29. Cooley CZ, McDaniel PC, Stockmann JP, et al. A portable scanner for magnetic resonance imaging of the brain. Nat Biomed Eng. 2021;5(3):229-239.

30. Ursino M, Giulioni M. Quantitative assessment of cerebral autoregulation from transcranial Doppler pulsatility: a computer simulation study. Med Eng Phys. 2003;25(8):655-666.

31. Zhao X, Yu Y, Xu W, et al. Apolipoprotein A1-unique peptide as a diagnostic biomarker for acute ischemic stroke. Int $J$ Mol Sci. 2016;17(4):458.

32. Tao L, ShiChuan W, DeTai Z, Lihua H. Evaluation of lipoprotein-associated phospholipase A2, serum amyloid $\mathrm{A}$, and fibrinogen as diagnostic biomarkers for patients with acute cerebral infarction. J Clin Lab Anal. 2020;34(3): e23084.

\section{Disclosures}

Dr. Mack: direct stock ownership in Integra, Cerebrotech, and Rebound Therapeutics.

\section{Author Contributions}

Conception and design: Shahrestani. Acquisition of data: Shahrestani, Wishart, Han. Analysis and interpretation of data: Shahrestani, Wishart, Han. Drafting the article: Shahrestani. Critically revising the article: Shahrestani, Strickland,

Bakhsheshian, Mack, Toga, Sanossian, Tai. Reviewed submitted version of manuscript: Shahrestani, Strickland, Bakhsheshian, Mack, Toga, Sanossian, Tai. Administrative/technical/material support: Zada, Tai. Study supervision: Zada, Tai. 
Shahrestani et al.

\section{Supplemental Information}

Videos

Video Abstract. https://vimeo.com/557536887.

\section{Correspondence}

Gabriel Zada: Keck School of Medicine, University of Southern California, Los Angeles, CA. gabriel.zada@med.usc.edu. 